\title{
HALF-CANONICAL SERIES ON ALGEBRAIC CURVES
}

\author{
MONTSERRAT TEIXIDOR I BIGAS
}

\begin{abstract}
Denote by $\mathscr{M}_{g}^{r}$ the locus in the moduli space of curves of genus $g$ of those curves which have a theta-characteristic of (projective) dimension at least $r$. We give an upper bound for the dimension of $\mathscr{M}_{g}^{r}$ and we determine this dimension completely for $r \leqslant 4$. For $r \leqslant 4$, we prove also that a generic point in every component of $\mathscr{M}_{g}^{r}$ has a single theta-characteristic of this dimension.
\end{abstract}

0. Introduction. Let $\mathscr{M}_{g}$ be the moduli space of smooth, complete curves of genus $g$ over the complex field $\mathbf{C}$. We investigate the subloci $\mathscr{M}_{g}^{r}$ of $\mathscr{M}_{g}$. These are defined as the loci of curves having a theta-characteristic (i.e. a line bundle $L$ such that $L \otimes L=K_{C}$ ) of (projective) dimension at least $r$ and of the same parity as $r$.

By Clifford's Theorem, it is clear that $\mathscr{M}_{g}^{r}$ is empty if $r>\frac{1}{2}(g-1)$. On the other hand, one can easily see that hyperelliptic curves have theta-characteristics of all dimensions $r$ with $0 \leqslant r \leqslant \frac{1}{2}(g-1)$.

In [H], Harris proves

(0.1) TheORem (CF. [H, THEOREM (1.10)]). Let $X \rightarrow S$ be a family of curves and $L$ a line bundle on $X$ such that the restriction $L(s)$ to every fiber $X(s)$ satisfies $L^{2}(s) \simeq$ $K_{X(s)}$. Then the subset of $S$

$$
\left\{s \in S \mid h^{0}(X(s), L(s)) \geqslant r+1 \text { and } h^{0}(X(s), L(s)) \equiv r+1(2)\right\}
$$

has codimension at most $\frac{1}{2} r(r+1)$ in $S$ at all of its points.

Combining this with the above facts, one obtains

(0.2) THeOREM (HARRIS). The locus $\mathscr{M}_{g}^{r}$ is empty if and only if $r>\frac{1}{2}(g-1)$. If $r \leqslant \frac{1}{2}(g-1)$, then any component of $\mathscr{M}_{g}^{r}$ has codimension at most $\frac{1}{2}(r+1)$ in $\mathscr{M}_{g}$.

One could ask if the lower bound of Harris for the dimension of the components of $\mathscr{M}_{g}^{r}$ is in fact an equality. This is not always the case, even for those components of $\mathscr{M}_{g}^{r}$ whose general point corresponds to a curve with a half-canonical series without fixed points, of dimension exactly $r$ and giving a birational morphism of $C$ in $\mathbf{P}^{r}$. A counterexample is provided, for instance, by the work of Accola $[\mathbf{A}]$ on curves of genus $3 r$ which possess a (necessarily half-canonical) simple series of dimension $r$.

Received by the editors June 16, 1986.

1980 Mathematics Subject Classification. Primary 14H10; Secondary 14H40, 14K25.

Key words and phrases. Theta-characteristic.

Partially supported by C.I.R.I.T. and Institut d'Estudis Catalans (treball baix contracta 6020-2224). 
In this case however $g$ is very small compared with $r$, as these are Castelnuovo extremal curves. In his paper, Harris asks whether the situation becomes regular when $g$ grows. Here we give an affirmative answer for $r \leqslant 4$.

In the first place, we find an upper bound for the dimension of the components of $\mathscr{M}_{g}^{r}$, namely $3 g-2 r+2$. This is sharp in the sense that for every $r$, there is one $g$ $(g=2 r+1)$, for which it is attained. In this case $\mathscr{M}_{g}^{r}$ is the hyperelliptic locus.

For $r=1$ and 2, the upper bound coincides with the lower bound of Harris. It follows from this that $\mathscr{M}_{g}^{2}$ has pure codimenion 3 in $\mathscr{M}_{g}$, as well as the classical result that $\mathscr{M}_{g}^{1}$ is a divisor in $\mathscr{M}_{g}$ (see $\left.[\mathbf{F}, \mathbf{B}]\right)$.

For $r \geqslant 3$, the upper bound is sharp only in the case mentioned above, namely $g=2 r+1$. As a consequence, $\mathscr{M}_{g}^{3}$ has codimension 6 in $\mathscr{M}_{g}$ when $g \geqslant 8$. For $r \geqslant 4$ the upper bound may be refined when $g \gg r$ and from this refinement the solution in case $r=4$ follows.

We show moreover that, for $r \leqslant 4$ and $g \gg r$, a generic point of a component of $\mathscr{M}_{g}^{r}$ has only one half-canonical series of this dimension which is simple and that for $r=1$ and 2 it has no fixed points.

The proof uses the deformation theory developed by Arbarello and Cornalba in [A, C1, 2] combined with some ideas inspired by recent work of Díaz [D].

I would like to thank Gerald Welters for his guidance during the preparation of this work and also Marc Coppens for pointing out some mistakes in a previous version of the manuscript.

1. Definitions and preliminaries. We recall first a few well-known facts and introduce notations that we are going to use throughout the paper.

In this work, $C$ will always denote a projective, nonsingular curve of genus $g$ defined over the complex field $\mathbf{C}$. If $F$ is a sheaf on $C$, the cohomology groups $H^{i}(C, F)$ will often be written $H^{i}(F)$. If $f: X \rightarrow S$ is a morphism of schemes, $X(s)$ will denote the fiber over $s \in S$. For any scheme $S$, $T_{S}(s)$ will mean the tangent space to $S$ at $s, T_{S}$ the tangent sheaf on $S$.

(1.1) With $C$ as above, there exist irreducible, nonsingular varieties $X, S, S$ quasiprojective and a flat projective morphism $p: X \rightarrow S$ such that

(a) Any fiber of $p$ is a nonsingular curve of genus $g$ and one of them is $C$.

(b) For every $s$ in $S$, the Kodaira-Spencer map $T_{S}(s) \rightarrow H^{1}\left(X(s), T_{X(s)}\right)$ is an isomorphism

(c) p has a section.

For such a family, there exists a Picard scheme $\operatorname{Pic}^{d}(X / S)$ (that we shall write $\mathrm{Pic}^{d}$ for short), together with a Poincare bundle on $X \times{ }_{S} \mathrm{Pic}^{d}$. This parametrizes line bundles of degree $d$ on the fibers of $p$.

There is also a scheme $G_{d}^{r}$ parametrizing linear series on the fibers of $p$ (see [A, C1, §2]). If $t$ is a point in $G_{d}^{r}$ corresponding to a curve $C$, a line bundle $L$ on $C$ and a subspace of dimension $r+1$ in $H^{0}(L)$, then there is an exact sequence ([A, C1, pp. 17-18])

$$
0 \rightarrow \operatorname{Hom}\left(W, H^{0}(L) / W\right) \rightarrow T_{G_{d}^{r}}(t) \rightarrow H^{1}\left(\Sigma_{L}\right) \rightarrow \operatorname{Hom}\left(W, H^{1}(L)\right)
$$


Here $\Sigma_{L}$ denotes the sheaf of differential operators of order at most one acting on $L$. The space $H^{1}\left(\Sigma_{L}\right)$ is naturally identified with $T_{\mathrm{Pic}^{d}}(L)$ and the last morphism is given by cup-product.

(1.3) Definition. We define a scheme $P$ by means of the following pull-back diagram

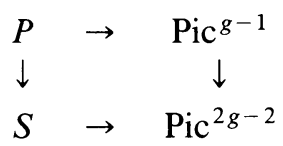

where the morphisms from $\mathrm{Pic}^{g-1}$ and $S$ to $\mathrm{Pic}^{2 \mathrm{g-2}}$ are obtained by means of the universal property of $\mathrm{Pic}^{2 g^{-2}}$ by using the square of the Poincare bundle and the dualizing sheaf respectively.

The scheme $P$ parametrizes curves of the family $p$ and theta-characteristics on them so it projects onto $S$ with degree $2^{2 g}$. It is known [M, Theorem, p. 184], that the parity of a theta-characteristic is locally constant. Therefore, $P$ decomposes into two parts $P^{0}$ and $P^{1}$ corresponding to even and odd theta-characteristics respectively.

We define $T^{r}$ by means of the pull-back diagram

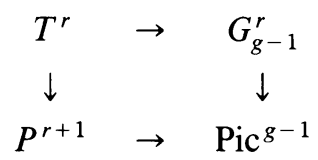

where the superindex in $P^{r+1}$ is understood modulo 2.

The scheme $T^{r}$ is closed in $G_{g-1}^{r}$ and parametrizes semicanonical series of dimension $r$ on $X \rightarrow S$ whose corresponding bundle $L$ satisfies $h^{0} L \equiv r+1$ (2). It projects onto $\mathscr{M}_{g}^{r} \cap h(S)$, where $h: S \rightarrow \mathscr{M}_{g}$ is the classifying morphism induced by $p$.

(1.4) Proposition. Let $g$ and $r$ satisfy $g>\frac{1}{2}\left(r^{2}+r+2\right)$. Let $M$ be a component of $\mathscr{M}_{g}^{r}$. Then a generic point $C$ of $M$ cannot be a covering of a curve of genus $g \geqslant 1$. Moreover, if $g>\frac{1}{2}\left(r^{2}+3 r+2\right), r \geqslant 2$, then $C$ has only simple half-canonical series of dimension $r$.

Proof. Assume the first statement false, i.e. $C$ is a covering of degree $t \geqslant 2$ of a curve of genus $g^{\prime} \geqslant 1$.

The curves of genus $g$ which are coverings of degree $t$ of some curve of genus $g^{\prime}$ depend on $2 g-2-(2 t-3)\left(g^{\prime}-1\right)$ moduli (see [L, Satz 1]). Therefore, by using (0.2), one finds

$$
2 g-2 \geqslant 2 g-2-(2 t-3)\left(g^{\prime}-1\right) \geqslant 3 g-3-\frac{1}{2} r(r+1)
$$

which contradicts the hypothesis on $g$.

Assume now that $g \geqslant \frac{1}{2}\left(r^{2}+3 r+2\right), r \geqslant 2$, and $C$ has a nonsimple semicanonical series of dimension $r$. So this series should give rise to a morphism in $\mathbf{P}^{r}$ which could be factored $C \rightarrow C^{\prime} \rightarrow \mathbf{P}^{r}$, where the first morphism has degree $t \geqslant 2$ and $C^{\prime}$ is a rational curve contained in no hyperplane of $\mathbf{P}^{r}$. This latter condition implies 
that the degree of $C^{\prime}$ is at least $r$, i.e. $\frac{1}{t}(g-1-k) \geqslant r$ where $k$ is the number of fixed points of the semicanonical series. Hence

$$
t \leqslant(1 / r)(g-1-k) \leqslant(1 / r)(g-1) .
$$

Moreover, as $C$ is a covering of degree $t$ of a rational curve, $M$ is contained in the set of $t$-gonal curves and one has the inequality of dimensions (cf. (0.2)) $2 g-2+2 t$ $-3 \geqslant 3 g-3-\frac{1}{2} r(r+1)$. Therefore

$$
t \geqslant \frac{1}{2}(g+2)-\frac{1}{4} r(r+1) .
$$

From (1.4a) and (1.4.b), in case $r>2$ one finds

$$
g \leqslant \frac{1}{2}\left(r^{2}+3 r+2\right)
$$

which contradicts the hypothesis.

In the case $r=2$, from (1.4.a) and (1.4.b) one finds $k=0, t=\frac{1}{2}(g-1)$. Hence $g$ is odd and for a generic point of the set of $t$-gonal curves of genus $g$ (which is irreducible of dimension $3 g-6$ ), the line bundle $L$ giving rise to the unique linear series of degree $t$ and dimension one satisfies $4 L=K$. This cannot be true: for a hyperelliptic curve $C$, consider the line bundle $L_{C}=L_{2} \otimes \mathcal{O}\left(\frac{1}{2}(g-5) P\right)$ where $L_{2}$ is the sheaf defining the $g_{2}^{1}$ and $P$ is not a Weierstrass point in $C$. Then $4 L_{C} \neq K$.

But by (3.1) and the irreducibility of the loci of special divisors on an hyperelliptic curve, one should be able to deform $L$ to $L_{C}$. This would imply that $4 L_{C}=K_{C}$ contradicting the choice of $L_{C}$.

The following lemma is implicit in [A, C2]. We include a proof here for the convenience of the reader.

(1.5) Lemma (Arbarello-Cornalba). Let $M$ be a subvariety of $\mathscr{M}_{g}$ of dimension at least $g, p: X \rightarrow U$ a family of curves such that the classifying map projects $U$ onto an open dense subset of $M$ and let

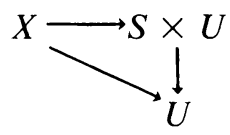

be a family of birational morphisms from the fibers of $p$ in a nonsingular algebraic surface $S$. Then, for a generic point $u$ in $U$, the normal bundle $N$ to the morphism $X(u) \rightarrow S$ satisfies $h^{1}(N)=0$.

Proof. The normal sheaf $N$ to $f$ is defined by means of the exact sequence

$$
0 \rightarrow T_{C} \rightarrow f^{*} T_{S} \rightarrow N \rightarrow 0 .
$$

Let $D$ be the ramification divisor of $f$ and $N^{\prime}$ the invertible rank one sheaf which fits in the exact sequence

$$
0 \rightarrow T_{C}(D) \rightarrow f^{*} T_{S} \rightarrow N^{\prime} \rightarrow 0 .
$$


There is a commutative diagram

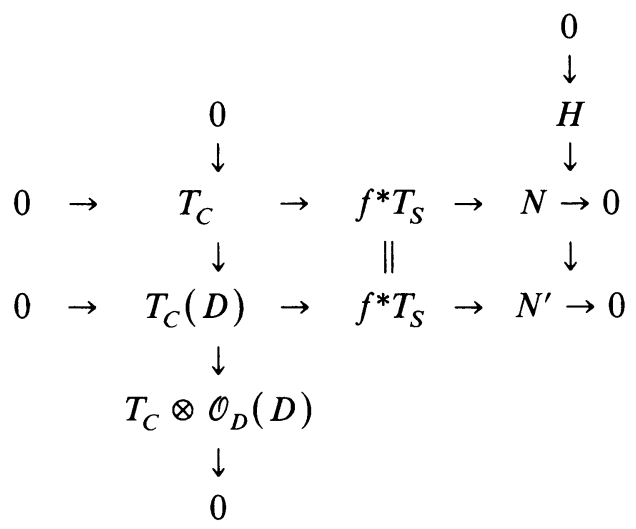

and clearly

$$
H \simeq T_{C} \otimes \mathcal{O}_{D}(D) \simeq \mathcal{O}_{D} ;
$$

let $t$ be a general point of $U$. Consider the Horikawa map $T_{U}(t) \rightarrow H^{0}(N)$ associated to the family of morphisms (1.5.1). By Lemma (1.4) in [A, C2], the image of this map intersects $H^{0}(H)$ in 0 and so it maps injectively in $H^{0}\left(N^{\prime}\right)$. Moreover, it is standard that the composition of the Horikawa morphism with the natural map $H^{0}(N) \rightarrow H^{1}\left(T_{C}\right)$ deduced from (1.5.2), is the Kodaira-Spencer map associated to $X \rightarrow U$ (see [Ho]). As $t$ is general in $U$, the dimension of the image of this map is at least $\operatorname{dim} M$. So, because of the hypothesis on $M, h^{0}\left(C N^{\prime}\right) \geqslant \operatorname{dim} M>g$. As $N^{\prime}$ is a line bundle on a curve of genus $g$, this implies that it is nonspecial and so $h^{1}\left(N^{\prime}\right)=0$. Then, from (1.5.3), (1.5.4) it follows that $h^{1}(N)=0$ as stated.

(1.6) Corollary. Let $g$ be at least 6. For a generic point $C$ of a component $M$ of $\mathscr{M}_{g}^{2}$ any half-canonical linear series of dimension 2 on $C$ is simple and gives rise to a morphism in $\mathbf{P}^{2}$ whose associated normal sheaf $N$ satisfies $h^{1}(N)=0$.

Proof. For $g=6$, if $L$ has fixed points, then $C$ is hyperelliptic and (0.2) contradicts the genericity of $C$. If $L$ has no fixed points, as $g-1=5$ is prime, $L$ is necessarily simple.

If $g \geqslant 7,(1.4)$ gives the first assertion.

Then use (0.2) and (1.5).

\section{Infinitesimal study of $T^{r}$ and applications.}

I. Some considerations about the tangent space to $T^{r}$.

Let $p: X \rightarrow S$ be a family of curves satisfying the conditions of (1.1). Let $t$ be a point in $T^{r}$ corresponding to a curve $C$, a theta-characteristic $L$ on $C$ and a subspace $W$ of dimension $r+1$ (of $H^{0}(L)$ ). Because of the definition of $T^{r}$ (cf. (1.3)), there is a pull-back diagram:

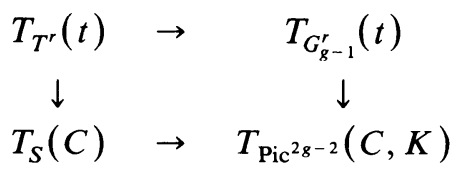


As the morphism of $\mathrm{Pic}^{g-1}$ in $\mathrm{Pic}^{2 g-2}$ given by tensor square is étale, it induces an isomorphism of tangent spaces. So, one finds

$$
H^{1}\left(\Sigma_{L}\right) \simeq T_{\mathrm{Pic}^{g-1}}(C, L) \simeq T_{\mathrm{Pic}^{2 g-2}}(C, K) \simeq H^{1}\left(\Sigma_{K}\right) .
$$

We recall that, by hypothesis (cf. (1.1.b)), $T_{S}(C)$ is isomorphic to $H^{1}\left(T_{C}\right)$. The image of $T_{S}(C)$ in $H^{1}\left(\Sigma_{K}\right)$ are those first order infinitesimal deformations $K_{\varepsilon}$ of $K$ which give the canonical sheaf on the corresponding deformation $C_{\varepsilon}$ of $C$, i.e. those deformations of $K$ which maintain the $g$ sections.

Therefore, by using (1.2), diagram (2.1) becomes

$$
\begin{aligned}
& 0 \\
& \downarrow \\
& \operatorname{Hom}\left(W, H^{0}(L) / W\right) \\
& 0 \rightarrow T_{T^{r}}(t) \rightarrow T_{G_{g-1}^{r}}(t) \\
& \downarrow \quad \downarrow \\
& 0 \rightarrow H^{1}\left(T_{C}\right) \rightarrow H^{1}\left(\Sigma_{L}\right) \simeq H^{1}\left(\Sigma_{K}\right) \rightarrow \operatorname{Hom}\left(H^{0}(K), H^{1}(K)\right) \rightarrow 0 \\
& \downarrow \\
& \operatorname{Hom}\left(W, H^{1}(L)\right)
\end{aligned}
$$

where the square is a pull-back diagram.

It can be checked that the isomorphism $H^{1}\left(\Sigma_{L}\right) \simeq H^{1}\left(\Sigma_{K}\right)$ sends a cocycle $\left(s_{i j}\right) \in H^{1}\left(\Sigma_{L}\right)$ to $\left(\mathrm{Id} \otimes s_{i j}+s_{i j} \otimes \mathrm{Id}\right) \in H^{1}\left(\Sigma_{K}\right)$. So, the composed map $T_{G_{g-1}^{r}}(t)$ $\rightarrow \operatorname{Hom}\left(H^{0}(K), H^{1}(K)\right)$ factors through $\operatorname{Hom}\left(H^{0}(K) / W \cdot W, H^{1}(K)\right)$ where $W \cdot W$ denotes the image of $W \otimes W$ in $H^{0}(K)$ by means of the Petri morphism.

One finds a diagram:

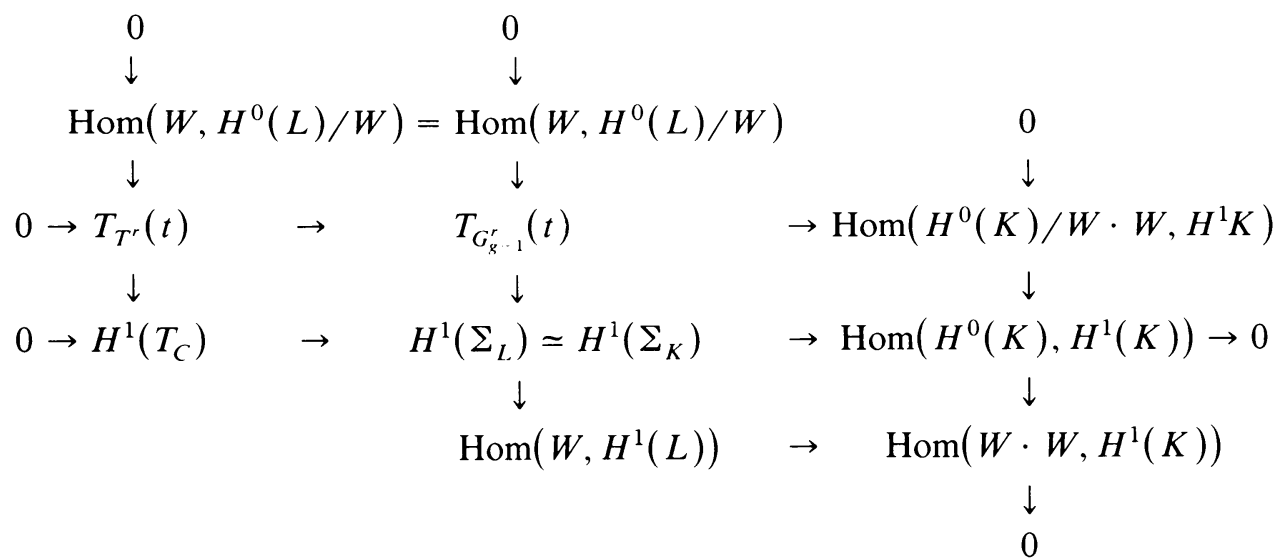

This is exact, exactness in the upper row being deduced from the fact that the left lower square is a pull-back.

We shall repeatedly consider the following situation:

(2.3) Let $t$ be a point of $T^{r}$ corresponding to a curve $C$, a theta-characteristic $L$ on $C$, and an $(r+1)$-dimensional subspace $W$ of $H^{0}(L)$. Let $D$ be the fixed part of the series corresponding to $W, k$ its degree, $q$ an equation for $D, L^{\prime}=L \otimes \mathcal{O}_{C}(-D), W^{\prime}$ the 
subspace of $H^{0}\left(L^{\prime}\right)$ whose image by the natural inclusion $\cdot q: H^{0}\left(L^{\prime}\right) \rightarrow H^{0}(L)$ is $W$. Let $t^{\prime}=\left(C, L^{\prime}, W^{\prime}\right)$ be the corresponding point in $G_{g-1-k}^{r}$. Denote by $f$ the morphism of $C$ in $\mathbf{P}^{r}$ associated to $W^{\prime}$.

Consider the following diagram (cf. [A, C1, (4.1)]):

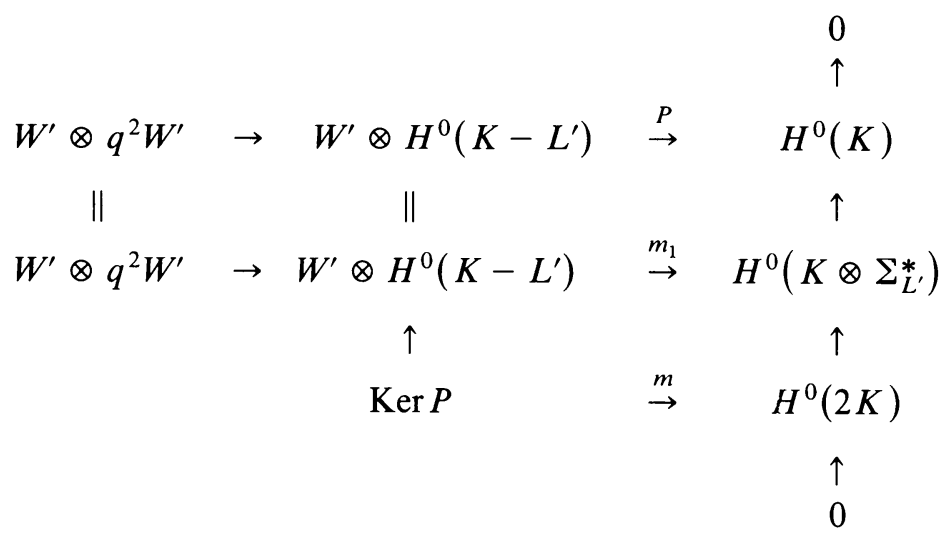

Here $P$ is the Petri morphism, the vertical sequence in the right is exact, $m_{1}$ is the dual of the natural contraction map and $m$ is obtained from $m_{1}$ by restriction.

Consider also the following diagram of exact sequences (cf. [A, C1, 5.1])

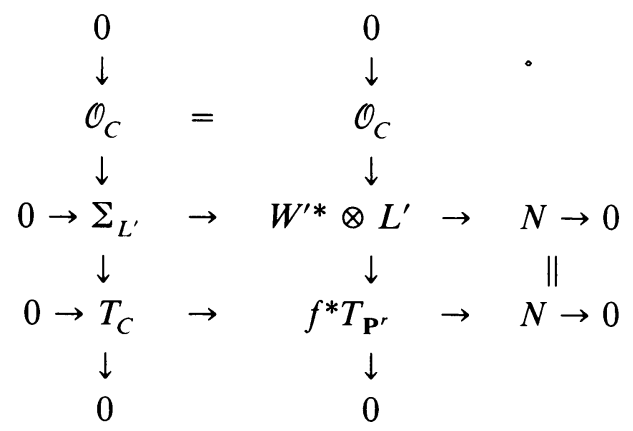

where $N$ is the normal sheaf to $f$, the vertical sequence in the middle is obtained by pulling-back to $C$ the Euler sequence in $\mathbf{P}^{r}$ and the morphism of $\Sigma_{L^{\prime}}$ in $W^{\prime *} \otimes L^{\prime}=$ $\operatorname{Hom}\left(W^{\prime}, L^{\prime}\right)$ is defined by contraction.

Taking homology one obtains:

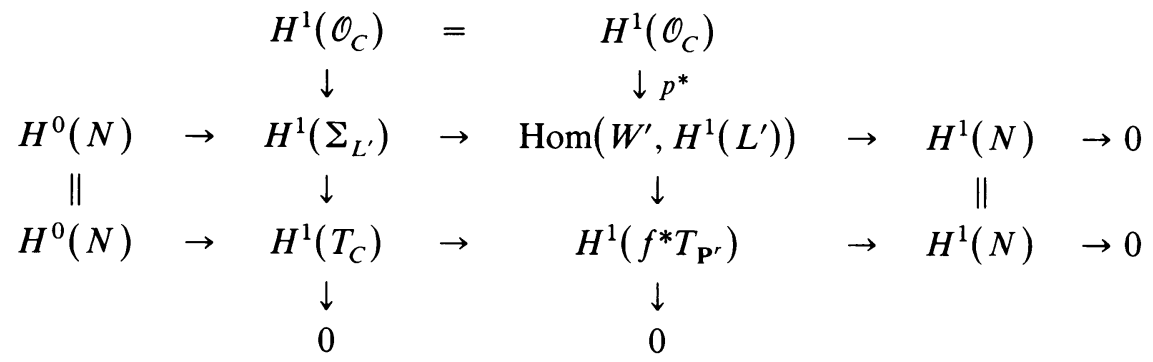


Therefore $H^{1}\left(f^{*} T_{\mathbf{P}^{r}}\right)$ is identified with the dual of $\operatorname{Ker} P$. Moreover, because of (1.2), the image of $T_{G_{z-1-k}^{r}}(t)$ in $H^{1}\left(\Sigma_{L^{\prime}}\right)=T_{\mathrm{Pic}^{g^{-1-k}}}\left(L^{\prime}\right)$ is the image of the morphism above from $H^{0}(N)$ to $H^{1}\left(\Sigma_{L^{\prime}}\right)$. So one obtains the following diagram [A, C1, p. 35]:

$$
\begin{array}{cccc}
T_{G_{g-1-k}^{r}}\left(t^{\prime}\right) \stackrel{h}{\rightarrow} H^{1}\left(\Sigma_{L^{\prime}}\right) & \rightarrow & \operatorname{Hom}\left(W^{\prime}, H^{1}\left(L^{\prime}\right)\right) & \rightarrow H^{1}(N) \rightarrow 0 \\
\| & \downarrow g_{L^{\prime}} & \downarrow & \| \\
T_{G_{g-1-k}^{r}}(t) \rightarrow H^{1}\left(T_{C}\right) \stackrel{m^{*}}{\rightarrow} & (\operatorname{Ker} P)^{*} & \rightarrow(\operatorname{Ker} m)^{*} \rightarrow 0 \\
\downarrow & \downarrow & \\
0 & 0 &
\end{array}
$$

Suppose now that the point $t$ corresponds to a complete theta-characteristic of dimension $r$, i.e. $W=H^{0}(L)$ and hence $W^{\prime}=H^{0}\left(L^{\prime}\right)$.

Assume that $t$ is a generic point of a component of $T^{r}$. Then, up to a finite base-change, there are $k$ sections $s_{1} \cdots s_{k}$ of $p: X \rightarrow S$ defined in a neighborhood of $C$ in $S$ such that, when restricted to the image of $T^{r}$ in $S$ they give rise to the fixed points of the theta-characteristic in the fibers of $p$.

One obtains then a commutative diagram:

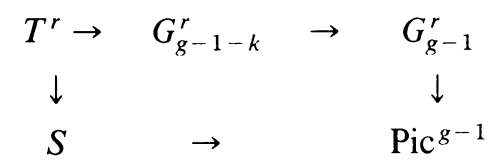

Taking tangent spaces, one has a factorization of one of the morphisms in (2.2)

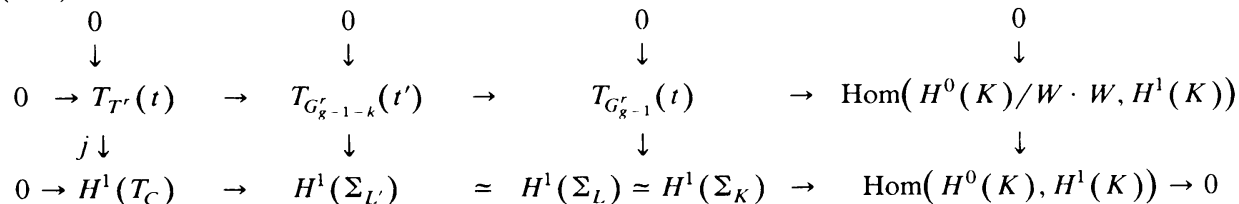

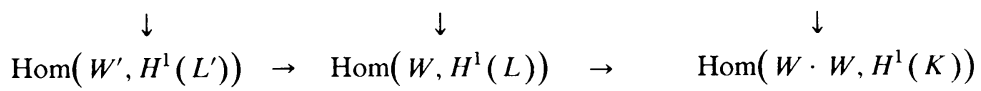

$$
\begin{aligned}
& H^{1}(N) \\
& \downarrow
\end{aligned}
$$

where the isomorphism $H^{1}\left(\Sigma_{L^{\prime}}\right) \simeq H^{1}\left(\Sigma_{L}\right)$ is the differential of the isomorphism from $\mathrm{Pic}^{g-1-k}$ to $\mathrm{Pic}^{g-1}$ given by tensor product with the sheaf

$$
\mathcal{O}_{x}\left(s_{1}(S)+\cdots+s_{k}(S)\right) \text {. }
$$

As the upper left rectangle is a pull-back, so is the upper left square.

From the commutative diagram

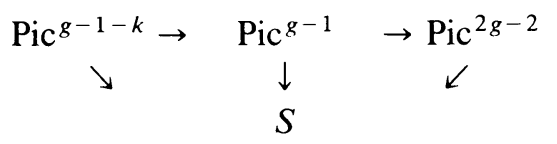


one obtains, by taking tangent spaces:

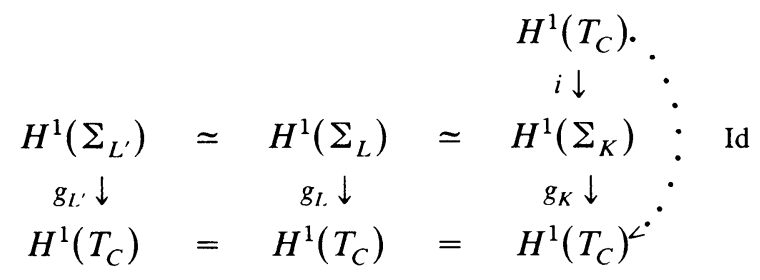

Therefore $i$ (interpreted through the isomorphisms in the upper row) is a section of $g_{L^{\prime}}$. Hence, with the notations of (2.5), (2.6)

$$
j\left(T_{T^{r}}(t)\right) \subset g_{L^{\prime}}\left(h\left(T_{G_{g-1-k}^{r}}(t)\right)\right)=\operatorname{Ker} m^{*}
$$

where the last equality follows from (2.5).

Then, one finds

$$
\begin{aligned}
\operatorname{dim} T^{r} & \leqslant \operatorname{dim} T_{T^{r}}(t) \leqslant \operatorname{dim} j\left(T_{T^{r}}(t)\right) \leqslant \operatorname{dim} \operatorname{Ker} m^{*} \\
& =3 g-3-\operatorname{dim} \operatorname{Ker} P+\operatorname{dim} \operatorname{Ker} m .
\end{aligned}
$$

We recall now that, by hypothesis, $2 L=K$, i.e. $K-L=L$. Therefore, if $s$ and $s^{\prime}$ are elements in $W^{\prime}, s \otimes q^{2} s^{\prime}-s^{\prime} \otimes q^{2} s$ belongs to $\operatorname{Ker} P$. In particular, $\operatorname{Ker} P$ has dimension at least $\frac{1}{2} r(r+1)$, as it contains the independent elements $s_{i} \otimes q^{2} s_{j}-s_{j}$ $\otimes q^{2} s_{i}, 0 \leqslant i \leqslant j \leqslant r$, for a basis $s_{i}$ of $W^{\prime}$.

(2.9) Definition. The elements of $\operatorname{Ker} P$ of the form $s \otimes q^{2} s^{\prime}-s^{\prime} \otimes q^{2} s$ will be called decomposable. The set of decomposable elements will be denoted by $G$ and its projectivization by $G^{\prime}$.

We point out that the point in $G^{\prime}$ coming from $s \otimes q^{2} s^{\prime}-s^{\prime} \otimes q^{2} s$ depends only on the one-dimensional linear subseries of $\left(L^{\prime}, W^{\prime}\right)$ generated by $s$ and $s^{\prime}$. In fact $G^{\prime}$ is isomorphic to the Grassmannian of lines in $\mathbf{P}^{r}$ canonically immersed in a linear subspace of dimension $\frac{1}{2} r(r+1)-1$ of the projectivization of Ker $P$.

(2.10) LemMA. Let $t$ be a generic point of a component of $T^{r}$ corresponding to a complete theta-characteristic of dimension $r$. Assume Ker $m$ intersects the linear span $\langle G\rangle$ of $G$ in $0(c f .(2.9))$. Then $\operatorname{dim} \operatorname{Ker} P=\frac{1}{2} r(r+1)+\operatorname{dim} \operatorname{Ker} m$ and the image $T_{T^{r}}(t)$ in $H^{1}\left(T_{C}\right)$ is the kernel of $m^{*}$.

Proof. As $\langle G\rangle \cap \operatorname{Ker} m=0, \operatorname{dim} \operatorname{Ker} P \geqslant \operatorname{dim}\langle G\rangle+\operatorname{dim} \operatorname{Ker} m=\frac{1}{2} r(r+1)+$ $\operatorname{dim} \operatorname{Ker} m$. Then, (2.8) gives

$\operatorname{dim} T^{r} \leqslant \operatorname{dim} \operatorname{Ker} m^{*} \leqslant 3 g-3-\operatorname{dim} \operatorname{Ker} P+\operatorname{dim} \operatorname{Ker} m \leqslant 3 g-3-\frac{1}{2} r(r+1)$.

On the other hand, by $(0.1), \operatorname{dim} T^{r} \geqslant 3 g-3-\frac{1}{2} r(r+1)$. Therefore all the inequalities are equalities and the result follows using (2.7).

II. Nonexistence of fixed points.

(2.11) Proposition. If the generic point of a component of $T^{r}$ is a complete half-canonical series of dimension $r$ such that the associated morphism $m$ (cf. (2.4)) satisfies $\operatorname{Ker} m=0$, then the half-canonical series has no fixed points. 
Proof. The hypothesis in (2.10) is satisfied. Therefore, taking into account that $(\operatorname{Ker} m)^{*}=H^{1}(N)$ (cf. (2.5)), diagram (2.6) may be completed to:

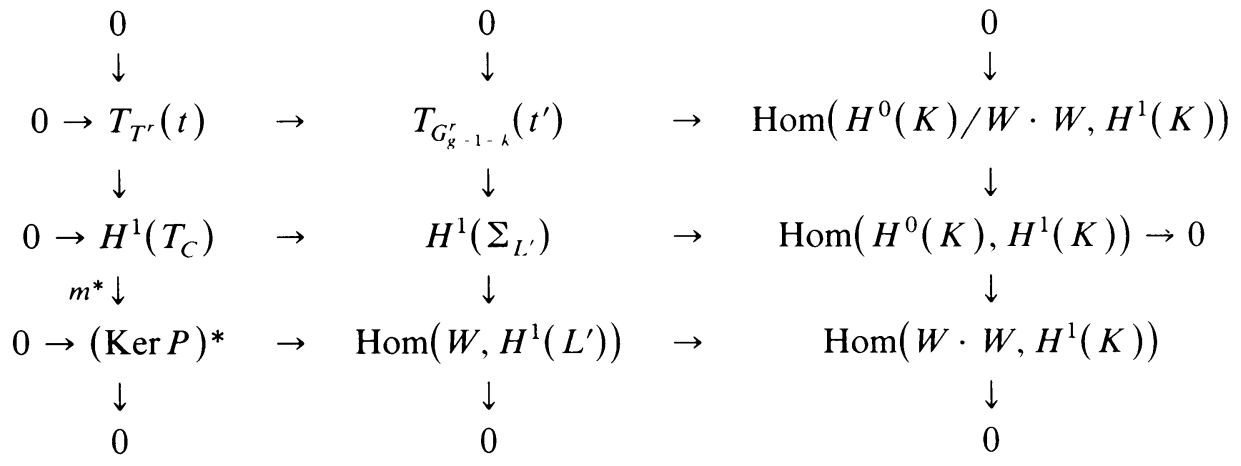

Here the 0 in the lower row is obtained by diagram chasing, using the fact that the upper left square is a pull-back.

By the Snake lemma and the exactness of the left column, coker $1=0$. By $(2.10)$, $\operatorname{dim} \operatorname{Ker} P=\frac{1}{2} r(r+1)$. Then, a computation using the first row and column gives

$$
\operatorname{dim} T_{G_{g-1-k}^{r}}\left(t^{\prime}\right)=4 g-3-(r+1)^{2} .
$$

From the central column

$$
\operatorname{dim} T_{G_{g-1-k}^{\prime}}\left(t^{\prime}\right)=4 g-3+(r+1) h^{1}\left(L^{\prime}\right)=4 g-3-(r+1)^{2}-k(r+1)
$$

where the last equality follows by Riemann-Roch because of the hypothesis of the series being complete. Therefore $k=0$.

III. An upper bound in the dimension of the components of $\mathscr{M}_{\mathrm{g}}^{r}$.

(2.12) Lemma. Completing notations in (2.3), let $V^{\prime}$ be a two-dimensional linear subspace of $W^{\prime}, F$ the fixed part of the corresponding one-dimensional series and $R$ the ramification divisor of the morphism $C \rightarrow \mathbf{P}^{1}$ it induces. Then, the image by $m$ ( $c f$. (2.4)) of the one-dimensional subspace of $\operatorname{Ker} P$ associated to this series (cf. (2.9)) is the one-dimensional linear space in $H^{0}(2 K)$ corresponding to the divisor $R+2 D+2 F$.

Proof. Choose a basis $a, b$ of $V^{\prime}$ so that $b$ has no multiple zeros outside $F$. Write $a=f b$ with $f$ a meromorphic function. Then $f$, as a morphism of $C$ in $\mathbf{P}^{1}$, is unramified at infinity.

By definition (cf. (2.4)), $m$ is obtained from $m_{1}$ by restriction and $m_{1}$ is the dual of the natural contraction

$$
H^{1}\left(\Sigma_{L^{\prime}}\right) \rightarrow \operatorname{Hom}\left(W^{\prime}, H^{1}\left(L^{\prime}\right)\right)=\operatorname{Hom}\left(W^{\prime} \otimes H^{0}\left(K-L^{\prime}\right), H^{1}(K)\right) .
$$

More explicitly, once an affine covering $U_{i}$ of $C$ has been chosen, any element in $H^{1}\left(\Sigma_{L^{\prime}}\right)$ is represented by a cocycle $\left(s_{i j}\right)$. Then $m_{1}^{*}\left(\left(s_{i j}\right)\right)(w)=\left(s_{i j}(w)\right) \in H^{1}\left(L^{\prime}\right)$ if $w \in W^{\prime}$ or $m_{1}^{*}\left(\left(s_{i j}\right)\right)(w \otimes \omega)=\left(s_{i j}(w) \omega\right) \in H^{1}(K)$ if $w \in W^{\prime}, \omega \in H^{0}\left(K-L^{\prime}\right)$.

In particular, if $v_{i j}$ is the derivation associated to $s_{i j}$, then

$$
m_{1}^{*}\left(s_{i j}\right)\left(a \otimes q^{2} b-b \otimes q^{2} a\right)=\left(s_{i j}(a) \cdot q^{2} b-s_{i j}(b) q^{2} a\right)=v_{i j}(f) q^{2} b^{2} .
$$


Therefore, the dual of the restriction of $m$ to the subspace $a \otimes q^{2} b-b \otimes q^{2} a$ operates as contraction with $f$

$$
\begin{aligned}
H^{1}\left(T_{C}\right) & \rightarrow\left\langle a \otimes q^{2} b-b \otimes q^{2} a\right\rangle^{*} \simeq H^{1}(K), \\
\left(v_{i j}\right) & \rightarrow v_{i j}(f) q^{2} b^{2} .
\end{aligned}
$$

Hence, $m\left(a \otimes q^{2} b-b \otimes q^{2} a\right)=(d f) q^{2} b^{2}$. Denote by $D_{a}$ and $D_{b}$ the divisors of zeros of $a$ and $b$ respectively.

By the choice of $a$ and $b, d f$ is a meromorphic differential whose divisor of zeros is $R$ and whose divisor of poles is twice the divisor of poles of $f$, i.e. $2\left(D_{b}-F\right)$. Therefore the divisor of $d f \cdot q^{2} b^{2}$ is

$$
R-2\left(D_{b}-F\right)+2 D+2 D_{b}=R+2 F+2 D
$$

as asserted.

(2.13) Theorem. Any component $M$ of $\mathscr{M}_{g}^{r}$ has dimension at most $3 g-2 r-2$. For $r \geqslant 3$ equality holds only for $g=2 r+1$ and in this case $M$ is the hyperelliptic locus. For $r \geqslant 4$ and $g \geqslant \max \left(12 r-22, \frac{1}{2}\left(r^{2}+3 r+2\right)\right)$, one has $\operatorname{dim} M \leqslant 3 g-4 r$ +3 .

(2.14) Corollary. For $r=3$ and $g \geqslant 8$ and for $r=4$ and $g \geqslant 26$, at a generic point of a component of $T^{r}$ projecting onto $M$, Ker $m \cap\langle G\rangle=0$ (cf. (2.9), (2.4) for the notations).

Proof of (2.13), (2.14). A general point of $M$ is a curve which has a complete semicanonical series of dimension $r+2 k, k \geqslant 0$. If $k>0, M$ is a component of $\mathscr{M}_{g}^{r+2 k}$. As the upper bound we try to prove is a decreasing function of $r$, we may assume $k=0$.

Let $T$ be a component of $T^{r}$ projecting onto $M$ and $t$ a generic point of $T$. By (2.12), the set $G$ defined in (2.9) cuts Ker $m$ in 0 . Hence $G^{\prime}$ does not intersect the linear subspace $L=\mathbf{P}(\operatorname{Ker} m)$. Therefore

$$
\operatorname{dim} \operatorname{Ker} m+\operatorname{dim} G^{\prime} \leqslant \operatorname{dim} \operatorname{Ker} P-1,
$$

As $G^{\prime}$ is a Grassmannian of lines in $\mathbf{P}^{r}$, it has dimension $(2 r-1)$. So,

$$
\operatorname{dim} \operatorname{Ker} m \leqslant \operatorname{dim} \operatorname{Ker} P-2(r-1)-1
$$

and (2.8) gives

(2.13.1) $\operatorname{dim} j\left(T_{T^{r}}(t)\right) \leqslant 3 g-3-\operatorname{dim} \operatorname{Ker} P+\operatorname{dim} \operatorname{Ker} m \leqslant 3 g-2 r-2$

which proves the first assertion in (2.13).

Assume now

(a) $r \geqslant 3, g>2 r+1$ and $\operatorname{dim} M=3 g-2 r-2$ or

(b) $r \geqslant 4, g \geqslant \max \left(\frac{1}{2}\left(r^{2}+3 r+2\right), 12 r-22\right)$, and $\operatorname{dim} M>3 g-4 r+3$.

Condition (a) implies that $L$ has the maximal dimension of a linear subspace in $\mathbf{P}$ not intersecting $G^{\prime}$. Therefore the linear space generated by $L$ and a generic point in $G^{\prime}$ intersects $G^{\prime}$ in other points.

Condition (b) implies (cf. (2.13.1)) that $\operatorname{dim} \operatorname{Ker} m \geqslant \operatorname{dim} \operatorname{Ker} P-4 r+7$. Therefore $L$ meets the variety of chords of $G^{\prime}$ (which has dimension $4 r-7$ ). 
In both cases there is a pair of points in $G$ having the same image by $m$. In case (a) one of the points in the pair (and hence also the other) may be assumed to be generic in $G$.

Using (2.12) we find two one-dimensional linear subseries $g_{1}, g_{2}$ of $(L, W)$ with fixed parts $F_{1}, F_{2}$ and ramification divisors $R_{1}, R_{2}$ such that

$$
R_{1}+2 F_{1}=R_{2}+2 F_{2} \text {. }
$$

Let $R$ be the greatest effective divisor contained in $R_{1}$ and $R_{2}$ and write $R_{i}=R+A_{i}$. Then $A_{1}+2 F_{1}=A_{2}+2 F_{2}$ and $A_{1}, A_{2}$ have no points in common, so $A_{2} \leqslant 2 F_{1}(2.13 .3)$.

Consider the morphism $\left(f_{1}, f_{2}\right): C \rightarrow \mathbf{P}^{1} \times \mathbf{P}^{1}$ obtained as the product of the two morphisms associated to the two one-dimensional linear series considered above.

This morphism is ramified at the points shared by the two ramification divisors of $f_{1}$ and $f_{2}$, i.e. at $R$. Hence, one finds a diagram defining $N^{\prime}$ (cf. (1.5.3)):

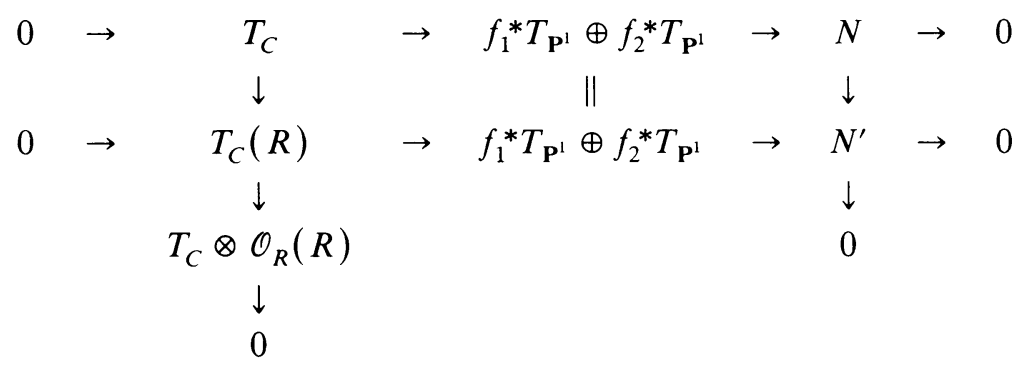

By Hurwitz's formula, $f_{i}^{*} T_{\mathbf{P}^{1}} \simeq T_{C}\left(R_{i}\right)$. Therefore, computing with the lower row, one finds

$$
N^{\prime} \simeq T_{C}\left(R_{1}\right) \otimes T_{C}\left(R_{2}\right) \otimes\left(T_{C}(R)\right)^{v}=T_{C}\left(R_{1}+A_{2}\right) .
$$

Hence

$$
h^{1}\left(N^{\prime}\right)=h^{0}\left(K-N^{\prime}\right)=h^{0}\left(2 K-R_{1}-A_{2}\right) \geqslant h^{0}\left(2 K-R_{1}-2 F_{1}\right) \geqslant 1
$$

where the first inequality comes from (2.13.3) and the last from the fact (see (2.12)) $R_{1}+2 F_{1}+2 D \equiv 2 K$.

As $\operatorname{dim} M>g$ and $h^{1}\left(N^{\prime}\right)=h^{1}(N)$, this means by (1.5) that the morphism $\left(f_{1}, f_{2}\right)$ is composed with an involution.

In case (b) (1.4) asserts that $C$ is not a covering of a curve of genus $g \geqslant 1$ and that the morphism $C \rightarrow \mathbf{P}^{r}$ induced by the half-canonical series, is simple.

In case (a) a proof as in (1.4) using the conditions $\operatorname{dim} M=3 g-2 r-2$ and $g>2 r+1$ gives the same result.

The morphism $f_{i}$ is obtained from a one-dimensional linear subseries of $\left(L, H^{0}(L)\right)$. Equivalently, $f_{i}$ is obtained by composing $f$ with a projection from a codimension 2 subspace $X_{i}$ in $\mathbf{P}^{r}$. The condition for $\left(f_{1}, f_{2}\right)$ to be composed with an involution of degree $k$ is that $f_{1}$ and $f_{2}$ should be composed with the same involution. This means that the intersection of the hyperplanes generated by $X_{i}$ and a generic point of $C$ contains $k-1$ further points. 
In case (a), by the genericity of $X_{1}$ and the principle of general position, this implies $k \leqslant r-1$. As the Hurwitz scheme of coverings of degree $r-1$ and genus $g$ of $\mathbf{P}^{1}$ has dimension $2 g+2(r-1)-2$, one obtains

$$
3 g-2 r-2+2(r-1)=\operatorname{dim} M+\operatorname{dim} G r\left(r-2, \mathbf{P}^{r}\right) \leqslant 2 g+2(r-1)-2
$$

so $r \geqslant g / 2$ which contradicts (a).

Consider now case (b). We are assuming that the morphism $\left(f_{1}, f_{2}\right)$ given by the two linear subseries $g_{1}, g_{2}$ of $\left(L, H^{0}(L)\right)$ considered above may be factored $C \stackrel{1}{\rightarrow} \mathbf{P}^{1} \rightarrow \mathbf{P}^{1} \times \mathbf{P}^{1}$ where the first morphism has degree $k$ and the second bidegree $\left(n_{1}, n_{2}\right)$ with $k n_{i} \leqslant g-1$. Hence the dimension of $M$ must be at most the dimension of the set of $k$-gonal curves, namely $2 g+2 k-5$.

Assume $n_{i} \geqslant 3$, then $k \leqslant \frac{1}{3}(g-1)$ and $\operatorname{dim} M \leqslant 2 g+\frac{2}{3}(g-1)-5$ which contradicts (b).

If $n_{1}=n_{2}=1$, then $R_{1}=R_{2}$ and by (2.13.2) $F_{1}=F_{2}$. So, $g_{1}=g_{2}$ which is not the case.

If $n_{1}=n_{2}=2$, then $g_{1}, g_{2}$ are one-dimensional subseries of $1^{*} H^{0}\left(\mathcal{O}_{\mathbf{P}^{1}}(2)\right)+F_{1}$ and $1^{*} H^{0}\left(\mathcal{O}_{\mathbf{P}^{1}}(2)\right)+F_{2}$ respectively. As, by hypothesis, the line bundle for both series is the same, one obtains $F_{1} \equiv F_{2}$. If $F_{1}=F_{2}$, because $1^{*} H^{0}\left(\mathcal{O}_{\mathbf{P}^{1}}(2)\right)$ is 3-dimensional, $g_{1}$ and $g_{2}$ would share a section. But this implies that the line in $\left\langle G^{\prime}\right\rangle$ joining the points corresponding to $g_{1}$ and $g_{2}$ is entirely contained in $G^{\prime}$. By assumption, the image by $m$ of the 2-plane of $G$ corresponding to this line is a line in $H^{0}(2 K)$, so $G$ would cut Ker $m$ and this contradicts (2.11).

If $F_{1} \neq F_{2}$, then $h^{0}\left(F_{1}\right) \geqslant 2$ and $C$ is $(g-1-2 k)$-gonal. Therefore, by (b) $2 g+2+2(g-1-2 k)-5>3 g-4 r+3,2 g+2+2 k-5>3 g-4 r+3$. But these inequalities are incompatible with (b).

If $n_{1}=1, n_{2}=2$, then $g_{1}=1^{*} H^{0} \mathcal{O}_{\mathbf{p}^{1}}(1)+F_{1}, g_{2} \subset 1^{*} H^{0} \mathcal{O}_{\mathbf{p}^{1}}(2)+F_{2}$; so $R_{2}=$ $R_{1}+A_{1}+A_{2}$ where $A_{i} \in 1^{*} H^{0} \mathcal{O}_{\mathbf{P}^{1}}(1)$ are the pull-back of the ramification points of the double covering $\mathbf{P}^{1} \rightarrow \mathbf{P}^{1}$. From (2.13.2) $A_{1}+A_{2}+2 F_{2}=2 F_{1}$. As $A_{1}, A_{2}$ have disjoint supports, this implies that all points in the support of $A_{i}$ have even multiplicity in $A_{i}$ and are in the support of $R_{1}$ and 1 is ramified over at most $2 g+k-2$ distinct points. Hence $C$ depends on at most $2 g+k-5$ moduli. As $k \leqslant \frac{1}{2}(g-1)$, this contradicts (b) and ends the proof of (2.13).

We point out that, in case $r=3$ and $g \geqslant 8$ and in case $r=4$ and $g \geqslant 26$, we have proved that Ker $m$ intersects the variety of chords of $G$ in 0 and in both cases this chordal variety coincides with the span $\langle G\rangle$ of $G$ in $\operatorname{Ker} P$. Hence (2.14) is also proved.

(2.15) COROLlaRY. Let $M$ be a component of $\mathscr{M}_{g}^{r}, C$ a generic point in $M$. If $r \leqslant 3$ or $r=4$ and $g \geqslant 38$, then $C$ has no half-canonical linear series of dimension greater than $r$.

Proof. Assume that $C$ has a semicanonical series of dimension greater than $r$. Then $M$ would be contained in a component of $\mathscr{M}_{g}^{r+1}$ or $\mathscr{M}_{g}^{r+2}$. So, by (2.13), $\operatorname{dim} M \leqslant 3 g-2 r-4$, and $\operatorname{dim} M \leqslant 3 g-17$ if $r=4$ and $g \geqslant 38$. But this contradicts $(0.2)$. 


\section{Uniqueness of the half-canonical series.}

(2.16) TheOREM. For $r=1, r=2$ and $g \geqslant 6, r=3$ and $g \geqslant 9$ or $r=4$ and $g \geqslant 38$, a generic point of any component $M$ of $\mathscr{M}_{g}^{r}$ has only one half-canonical series of dimension $r$.

ProOf. Let $M$ be a component of $\mathscr{M}_{g}^{r}$ with $g$ and $r$ satisfying the hypothesis. Let $C$ be a generic point in $M$. From (2.15), a half-canonical linear series of dimension $r$ on $C$ is complete. Assume $C$ had two of them, then they would correspond to two different line bundles $L_{1}$ and $L_{2}$ on $C$. Let $t_{1}$ and $t_{2}$ be the corresponding points in $T^{r}$. By the genericity of $C$ in $M$ and the fact that the image in $H^{1}\left(T_{C}\right)$ of the tangent spaces to $T^{r}$ at both points has dimension equal to the dimension of $M$ (cf. (2.8), (2.10), (2.14)), these images must be the same. Moreover they are the kernels of the corresponding morphisms $m=m_{L_{r}}$ (cf. (2.10)). By duality, the images of the morphisms $m_{L}$ are also the same.

From (2.5) $(\operatorname{Ker} m)^{*}$ is identified with $H^{1}(N)$ and this is zero when $r=1$ and also when $r=2$ (cf. (1.6)). When $r=3$ or 4 , Ker $m$ intersects the space $\left\langle G_{L_{i}}\right\rangle$ in 0 (cf. (2.14)).

For $r=1$ or $2,\left\langle G_{L_{i}}\right\rangle=G_{L_{i}}$. For $r=3$ (resp. 4), $G_{L_{i}}$ has dimension 4 (resp. 6) and $\left\langle G_{L_{i}}\right\rangle$ has dimension 6 (resp. 10). As $\left\langle G_{L_{i}}\right\rangle \cap \operatorname{Ker} m=0$, these are also the dimensions of the images of $G_{L_{1}}$ and $\left\langle G_{L_{t}}\right\rangle$ by $m$. Therefore $G_{L_{1}}$ and $G_{L_{2}}$ must intersect. By (2.12), this means that there are one-dimensional linear subseries of $\left(L_{i}, H^{0}\left(L_{i}\right)\right)$ such that the corresponding fixed parts $F_{i}$ and ramification divisors $R_{i}$ satisfy

$$
R_{1}+2 F_{1}+2 D_{1}=R_{2}+2 F_{2}+2 D_{2}
$$

where $D_{i}$ denotes the fixed part of $\left(L_{i}, H^{0}\left(L_{i}\right)\right)$. Moreover for $r=1$ or 2 the one-dimensional series may be assumed to be generic in $H^{0}\left(L_{i}\right)$, so $F_{i}=0$.

This pair of linear series gives rise to the morphism $\left(f_{1}, f_{2}\right): C \rightarrow \mathbf{P}^{1} \times \mathbf{P}^{1}$ ramified over the divisor $R$ of points shared by $R_{1}$ and $R_{2}$. All we need to prove is that this morphism is birational. Then the proof is finished as in (2.13) by application of (1.5).

By $(1.4),\left(f_{1}, f_{2}\right)$ is not composed with a nonrational involution.

In case $r=1$ and 2, $D_{1}=D_{2}=0$ by (2.11) and we found already $F_{1}=F_{2}=0$. If the morphism were composed with a rational involution, then $L_{1}=L_{2}$ contradicting the hypothesis.

We study now the case $r=3$, the case $r=4$ being similar will be left to the reader. Assume $\left(f_{1}, f_{2}\right)$ could be factored as $C \stackrel{1}{\rightarrow} \mathbf{P}^{1} \rightarrow \mathbf{P}^{1} \times \mathbf{P}^{1}$ where 1 has degree $k$ and the second morphism has bidegree $\left(n_{1}, n_{2}\right)$.

$$
\text { If } \begin{aligned}
n_{1}=n_{2}=1 \text {, then } R_{1}=R \text { and from }(2.16 .1) F_{1}+D_{1}=F_{2}+D_{2} . \text { As } \\
\qquad L_{i}=1 * \mathcal{O}_{\mathbf{P}^{\prime}}(1)+F_{i}+D_{i},
\end{aligned}
$$

this gives $L_{1}=L_{2}$ and contradicts the hypothesis.

If $n_{1}=1, n_{2} \geqslant 2$, then $R_{2}=R_{1}+\sum A_{i}$ where each $A_{i}$ is the divisor of a fiber of $L$ and there are at least two different $A_{i}$ in the summation. Now (2.16.1) is

$$
2 F_{1}+2 D_{1}=\sum A_{i}+2 F_{2}+2 D_{2}
$$


therefore all points in $A_{i}$ are counted with multiplicity at least 2 and so they appear in the ramification divisor of 1 . It follows that the set of coverings of $\mathbf{P}^{1}$ such as 1 depend on at most $2 g+k-5$ moduli. As $k \leqslant \frac{1}{2}(g-1)$ and $\operatorname{dim} M \geqslant 3 g-9$, this implies $g \leqslant 9$ and in this case $k=4$. Now for $g=9$, one would have $L_{2}=1^{*} \mathcal{O}_{\mathbf{p}^{1}}(2)$. Therefore $C$ would be contained in a quadric cone in $\mathbf{P}^{3}$. But this is impossible by a moduli count (cf. (0.2) and [A, C3, Lemma (3.13)]).

If $n_{1}, n_{2} \geqslant 2$, then $L_{i}=\pi^{*} \mathcal{O}_{\mathbf{P}^{1}}(2)+T_{i}$ where $T_{i}$ is effective. As $L_{1} \neq L_{2}$ and $2 L_{1}=K=2 L_{2}, T_{1} \neq T_{2}$ and $2 T_{1} \equiv 2 T_{2}$. So $C$ is $2(g-1-2 k)$-gonal. This implies $2 g-5+4(g-1-k) \geqslant 3 g-9$. As $C$ is also $k$-gonal, one finds $2 g-5+$ $2 k \geqslant 3 g+9$. Hence $k \geqslant(g-4) / 2$. This inequality together with the preceding one gives $g \leqslant 10$ and if $g=10$ for all trigonal curves satisfy $6 g_{3}^{1}=K$ which is not the case (see the proof of (1.4)). The case $g=9$ may be discarded as above.

\section{Conclusions for $r \leqslant 4$.}

(2.17) Theorem. The locus $\mathscr{M}_{g}^{1}$ (resp. $\mathscr{M}_{g}^{2}$ ) has pure codimension 1 (resp. 3) in $\mathscr{M}_{g}$ if $g \geqslant 3$ (resp. $g \geqslant 5)$ and a generic point of any of its components is a curve which has only one half-canonical series of dimension 1 (resp. 2 if $g \geqslant 6$ ). Moreover this half-canonical series is not composed with an involution (resp. if $g \geqslant 6$ ) and has no fixed points.

Proof. The dimensionality statement follows from (0.2) and (2.13). The uniqueness of the half-canonical series from (2.16).

For $r=1$, (1.4) says that the series cannot be composed with a nonrational involution. If it were composed with a rational involution, then the dimension of the series would be at least 2, contradicting (2.15). For $r=2$ the simplicity of the series is contained in (1.4).

From (2.5), the condition Ker $m=0$ is equivalent to $h^{1}(N)=0$. From (1.6) this is satisfied for $r=2$ and it is obviously satisfied for $r=1$. Then (2.11) gives the nonexistence of fixed points.

We have obtained similar results for $r=3$ and 4 that we sum up in the following theorem. We point out however that the bounds given on the genus for $r=4$ are not the best possible and could be improved by ad hoc methods.

(2.18) TheOREM. The locus $\mathscr{M}_{g}^{3}$ (resp. $\mathscr{M}_{g}^{4}$ ) has pure codimension 6 (resp. 10) if $g \geqslant 8$ (resp. 26). If $g \geqslant 9$ (resp. $g \geqslant 38$ ), a generic point in a component of this locus has only one half-canonical series of dimension 3 (resp. 4) and this gives rise to a birational morphism in $\mathbf{P}^{3}$ (respectively $\mathbf{P}^{4}$ ).

3. Appendix. Irreducibility of $G_{d}^{1}$. We include a proof of this fact here because we have not been able to find a proper reference in the literature. Denote by $\mathscr{M}_{g, d}^{1}$ the locus of $d$-gonal curves in $\mathscr{M}_{g}$.

(3.1) Let $p: X \rightarrow S$ be a family as in (1.1). Choose a d such that $\rho(g, d, 1)=2 d-$ $g-2<0$. Then there is a single component of $G_{d}^{1}$ over every component of the pull-back of $\mathscr{M}_{g, d}^{1}$ to $S$. 
Proof. We shall assume that this is not the case for a certain component $M$ of the pull-back of $\mathscr{M}_{g, d}^{1}$ to $S$ and reach a contradiction.

(3.2) It is known that $G_{d}^{1}$ is nonsingular and has dimension $2 d+2 g-5$ if $g \geqslant 2$ (cf. [A, C1, p. 35]).

As the set of $d$-gonal curves $\mathscr{M}_{g, d}^{1}$ is irreducible of dimension $2 g+2 d-5$ if $g \geqslant 2$ and a generic $d$-gonal curve has only one linear series $g_{d}^{1}$ [A, C2, Theorem 2.6], there is exactly one component of $G_{d}^{1}$ projecting onto $M$.

Consider a component $G$ of $G_{d}^{1}$ projecting to but not onto $M$. We claim that a generic point of $G$ is a linear series without fixed points. Otherwise if $k$ were the number of fixed points of a generic series in $G$, then $\operatorname{dim} G=\operatorname{dim} G_{d-k}^{1}+k$ and this contradicts (3.1).

Replace $S$ by the image of $G$ in the $S$ above by means of the natural map and denote by $q$ the morphism $G \stackrel{q}{\rightarrow} S$. Now $\operatorname{dim} S \leqslant \operatorname{dim} M-1 \leqslant 2 g+2 d-6$ and therefore, by (3.2), the dimension of the fibers of $q$ is $a \geqslant 1$.

Write $G \times{ }_{S} G=\bigcup T_{i}$, the irreducible components of the product. The fibers of the surjective morphism $G \times{ }_{S} G \rightarrow S$ are the product of the fibers of $q$, hence their generic dimension is $2 a$. Therefore there is one $T_{i}$, say $T$, projecting onto $S$ with generic fiber of dimension $2 a$. Consider the pull-back diagram:

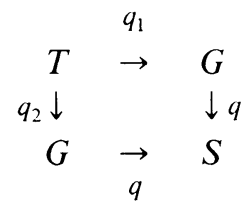

As $T$ projects onto $S$, so does the image of $T$ by $q_{2}$. Therefore the dimension of the generic fiber of $q_{1}$ is at most the dimension of the fibers of $q$. Hence,

$$
\begin{aligned}
\operatorname{dim} S+2 a & =\operatorname{dim} T \leqslant \operatorname{dim} q_{1}(T)+\operatorname{dim} \text { fiber } q_{1} \leqslant \operatorname{dim} q_{1}(T)+\operatorname{dim} \text { fiber } q \\
& =\operatorname{dim} q_{1}(T)+a \leqslant \operatorname{dim} G+a=\operatorname{dim} S+2 a .
\end{aligned}
$$

Hence $q_{1}(T)=G$ and similarly $q_{2}(T)=G$ and a generic point of $T$ corresponds to a pair of linear series which have no fixed points (as this happens for the generic point in $G$ ). Moreover, as $\operatorname{dim} T=G+2 a>\operatorname{dim} G, T$ is not contained in the diagonal of $G \times G$ and the two linear series in the pair are different.

Consider the morphism $f: C \rightarrow \mathbf{P}^{1} \times \mathbf{P}^{1}$ associated to this pair of linear series.

Assume $f$ were birational. Then, by [A, C2, Proposition (2.4)], $\operatorname{dim} T=g+4 d-7$. Hence $\operatorname{dim} G=g+4 d-7-a$. This, together with (3.2), gives $1 \leqslant a=2 d-g-2$ contradicting the hypothesis $\rho<0$.

Therefore $f$ is composed with an involution, i.e., $f$ may be factored $C \stackrel{1}{\rightarrow} C^{\prime} \rightarrow$ $\mathbf{P}^{1} \times \mathbf{P}^{1}$ where 1 has degree $m \geqslant 2, C^{\prime}$ has genus $g^{\prime}$ and the two rulings of $\mathbf{P}^{1} \times \mathbf{P}^{1}$ cut linear series on $C^{\prime}$ of degree $d / m$ whose pull-back to $C$ are the two series on $C$ considered above. Hence

$$
\operatorname{dim} T \leqslant \operatorname{dim} T^{\prime}+\left[2 g-2-m\left(2 g^{\prime}-2\right)\right]
$$

where $T^{\prime}$ is a component of $G_{d / m}^{1} \times G_{d / m}^{1}$ for curves of genus $g^{\prime}$ whose general point gives rise to a birational morphism of $C^{\prime}$ in $\mathbf{P}^{1} \times \mathbf{P}^{1}$ and the second summand is the number of moduli of an $m$-cover of $C^{\prime}$ of degree $g$. 
If $g^{\prime} \geqslant 2$, by [A, C2, Proposition (2.4)] $\operatorname{dim} T^{\prime}=g^{\prime}+4 d / m-7$.

Hence $\operatorname{dim} G=\operatorname{dim} T-a \leqslant 2 g+(1-2 m)\left(g^{\prime}-1\right)+4 d / m-9<2 g+2 d-$ 5 and this contradicts (3.2).

If $g^{\prime}=0, C^{\prime}=\mathbf{P}^{1}$. Then $m<d$ because, otherwise $g_{d}^{1}=\pi^{*} \mathcal{O}_{\mathbf{P}^{1}}(1)=h_{d}^{1}$.

Hence

$\operatorname{dim} G \leqslant \operatorname{dim} G_{m}^{1}+\operatorname{dim} G_{d / m}^{1}\left(\mathbf{P}^{1}\right)=2 g+2 m-5+2(d / m-1)<2 g+2 d-5$.

Similarly, if $g^{\prime}=1, C^{\prime}$ is elliptic and,

$$
\operatorname{dim} G \leqslant \operatorname{dim} m_{1}+\operatorname{dim} G_{d / m}^{1}\left(C^{\prime}\right)+2 g-2=2 g+2 d / m<2 g+2 d-5 .
$$

In both cases this contradicts (3.2).

\section{REFERENCES}

[A] R. Accola, Plane models for Riemann surfaces admitting certain half-canonical linear series, Part I, Riemann Surfaces and Related Topics, Proc. 1978 Stony Brook Conference, Editors, I. Kra and B. Maskit, Princeton Univ. Press, Princeton, N. J., 1981.

[A, C1] E. Arbarello and M. Cornalba, Su una congettura di Petri, Comment. Math. Helv. 56 (1981), $1-38$.

[A, C2] _ Footnotes to a paper of Benianino Segre, Math. Ann. 256 (1981), 341-362.

[A, C3] _ A few remarks about the variety of irreducible plane curves of given degree and genus, Ann. Sci. École Norm. Sup. (4) 16 (1963), 467-488.

[B] A. Beauville, Prym varieties and the Schottky problem, Invent. Math. 41 (1977), 149-196.

[D] S. Díaz, Tangent spaces in moduli via deformations with applications to Weierstrass points, Duke Math. J. 1 (1984).

[F] H. Farkas, Special divisors and analytic subloci of Teichmüller space, Amer. J. Math. 88 (1966), $881-901$.

[H] J. Harris, Theta characteristics on algebraic curves, Trans. Amer. Math. Soc. 271 (1982), 611-638.

[Ho] E. Horikawa, On deformations of holomorfic maps. I, J. Math. Soc. Japan 3 (1973), 373-396.

[L] H. Lange, Kurven mit rationaler Abbildung, Crelle's J. 295 (1977), 80-115.

[M] D. Mumford, Theta-characteristics on an algebraic curve, Ann. Sci. École Norm. Sup. (4) 4 (1971), $181-192$.

Facultat de Matematiques, Universitat de Barcelona, Gran Via 585, 08007 - Barcelona, SPAIN 